\title{
Modelo de competitividad de las empresas operadoras de telefonía móvil en México
}

Hugo Romo Vázquez

European Institute of

Management

hugoromovazquez@mail.telcel.com

Luis Arturo Rivas Tovar Escuela Superior de Comercio y Administración larivas33@hotmail.com

\section{Resumen}

El artículo presenta un modelo que explica la medida en que los recursos tangibles, el precio, la calidad, la productividad y la tecnología impactan en la competitividad de las empresas operadoras de telefonía móvil en México. El método empleado para ello fue hipotético deductivo con enfoque transversal y explicativo. La estrategia de recolección de información se basó en la aplicación de un cuestionario a cada una de las empresas operadoras; adicionalmente, se realizaron consultas a fuentes secundarias de información para complementar la recolección. Para alcanzar el objetivo de investigación se realizaron diferentes cálculos como análisis univariable, análisis factorial, análisis de correlación y análisis de regresión múltiple. El resultado principal de investigación fue un modelo causal expresado en dos diferentes funciones de regresión múltiple: en la primera función se obtuvo que el precio fue el único factor que explica la competitividad en términos de su rentabilidad; en la segunda regresión, la calidad y la productividad fueron los factores que explicaron la competitividad en términos de posición de mercado. Finalmente, los resultados de esta investigación permitieron dar algunas conclusiones respecto a las perspectivas teóricas y al sector móvil mexicano.

Palabras clave: competitividad, operadores móviles, México, modelos de competitividad, telecomunicaciones. 


\title{
Competitiveness model in mobile telephone enterprises in México
}

\begin{abstract}
This paper presents a model that explains how Mexican mobile operators' competitiveness was impacted by physical resources, price, quality, productivity and technology. The method for this research was a hypothetic and deductive one with transversal and explanatory characteristics. The strategy for data collection was the application of a questionnaire to all operators. In addition, different consultations were made by the authors from secondary sources in order to fill the data. So as to fulfill the research objective, different statistics analyses were made such as descriptive, correlation, factor and multiple regression analyses. The main result was the causal model expressed in terms of two different mathematic functions. In the first function, competitiveness was explained by the price. In the second, it was explained by quality and productivity. Finally, the research results allow to give some conclusion about the theory perspectives and the Mexican mobile sector.
\end{abstract}

Keywords: competitiveness, mobile operators, México, models, telecommunications.

\section{Introducción}

Se entiende por competitividad a la capacidad de una organización pública o privada, lucrativa o no, de mantener sistemáticamente ventajas que le permitan alcanzar, sostener y mejorar una determinada posición en el entorno socioeconómico (Hernández, 1999). Como disciplina de estudio, es un concepto desarrollado recientemente, aunque su aplicación práctica es antigua y universal. Existen diversos enfoques de análisis (sistémico, basados en el mercado, basados en la tecnología y basados en la innovación, entre otros) y diversos marcos de aplicación (nacional, sectorial y empresarial).

Si bien se asocia competitividad con la productividad, es necesario enfatizar que una empresa puede ser productiva y no ser competitiva, aunque para que una empresa sea competitiva sí debe ser productiva. Esto implica que la competitividad es un concepto más complejo y para fines prácticos cabría afirmar que la productividad es uno de sus componentes.

Para aclarar un poco el complejo bosque semántico de la competitividad es necesario iniciar este trabajo señalando que existen tres tipos de modelos para medir la competitividad: los que miden la competitividad en el nivel país, los que miden 
la competitividad de sectores industriales y los que miden la competitividad en empresas (Rivas, 2010). Por su parte, Cuervo (1993), Fernández (1993) y Salas (1993) coinciden en afirmar que el conjunto de factores explicativos de la competitividad de las empresas se puede estructurar en estas tres categorías:

a) Factores macroeconómicos, relacionados con el marco económico general.

b) Factores sectoriales, que pueden afectar a las empresas en función de la diferente naturaleza de su actividad económica.

c) Factores empresariales, que tienen que ver con las características intrínsecas de las propias empresas

\section{a) Factores macroeconómicos}

Desde esta perspectiva, el funcionamiento empresarial está condicionado por el entorno económico general en el que participa la empresa, el cual es común para cualquier otra unidad empresarial de su misma realidad económica y social, independientemente de su cometido productivo concreto, y que queda asociado al ámbito económico de cada país. De este modo, la competitividad se entiende como un problema de relación comparativa entre las empresas de los diferentes países y, desde el punto de vista del modelo neoclásico del comercio internacional, puede valorarse a partir del grado de participación de un país en la oferta mundial de bienes y servicios, así como de su precio relativo (Martin, 1993).

Como indica Salas (1993), desde este punto de vista, la discrecionalidad de las empresas es muy reducida al verse obligadas, para garantizar su supervivencia, a actuar siempre al límite de sus posibilidades de acuerdo con lo exigido por una situación de competencia en el sector y no únicamente de las condiciones que establezcan las variables macroeconómicas.

Desde este marco económico general, existen cinco tipos de políticas macroeconómicas que pueden ejercer una notable influencia sobre el comportamiento de la competitividad en las empresas (Segura, 1992): la política cambiaria que fija la paridad de la moneda, la política de rentas en la que se fijan los niveles salariales, la política monetaria, la política presupuestal y la política fiscal.

La configuración del gasto público es decisiva en la generación de un entorno favorable para la mejora de la competitividad a través de la realización de inversiones en infraestructura, transporte, comunicaciones, y también por medio de 
actividades conducentes a la creación de activos intangibles tales como la formación y capacitación de personal a través de la política educativa o el desarrollo tecnológico.

En el cuadro 1 se puede revisar, de una manera rápida, un pequeño resumen de los modelos de factores macroeconómicos más representativos que inciden en la competitividad.

\section{Cuadro 1}

Modelos de medición de la competitividad por país

\begin{tabular}{|c|c|c|c|c|}
\hline $\begin{array}{l}\text { Modelo } \\
\text { diamante de } \\
\text { Porter }\end{array}$ & $\begin{array}{l}\text { Modelo world } \\
\text { econcomic } \\
\text { forum }\end{array}$ & Modelo IMD & $\begin{array}{l}\text { Modelo } \\
\text { heritage } \\
\text { foundation }\end{array}$ & $\begin{array}{l}\text { Modelo } \\
\text { banco } \\
\text { mundial }\end{array}$ \\
\hline $\begin{array}{l}\text { Estrategia, } \\
\text { estructura y } \\
\text { rivalidad }\end{array}$ & Instituciones & $\begin{array}{l}\text { Actuación económica } \\
\text { (economía doméstica, } \\
\text { comercio internacional, } \\
\text { inversión internacional, } \\
\text { empleo y precios) }\end{array}$ & $\begin{array}{l}\text { Índice de } \\
\text { libertad } \\
\text { económica }\end{array}$ & $\begin{array}{l}\text { Número } \\
\text { de } \\
\text { trámites } \\
\text { necesarios }\end{array}$ \\
\hline $\begin{array}{l}\text { Comportamiento } \\
\text { de la demanda }\end{array}$ & Infraestructura & $\begin{array}{l}\text { Eficiencia del } \\
\text { gobierno(finanzas } \\
\text { públicas, política fiscal, } \\
\text { estructura institucional, } \\
\text { legislación empresarial), } \\
\text { estructura social }\end{array}$ & $\begin{array}{l}\text { Política } \\
\text { comercial } \\
\text { Carga fiscal }\end{array}$ & $\begin{array}{l}\text { Tiempo } \\
\text { dedicado } \\
\text { a cada } \\
\text { trámite }\end{array}$ \\
\hline $\begin{array}{l}\text { Servicios } \\
\text { conexos y de } \\
\text { apoyo }\end{array}$ & Macroeconomía & $\begin{array}{l}\text { Eficiencia empresarial } \\
\text { (productividad, mercado } \\
\text { laboral, finanzas, } \\
\text { prácticas de gestión y } \\
\text { actitudes y valores) }\end{array}$ & $\begin{array}{l}\text { Política } \\
\text { monetaria }\end{array}$ & $\begin{array}{l}\text { Costo } \\
\text { oficial } \\
\text { de cada } \\
\text { trámite }\end{array}$ \\
\hline $\begin{array}{l}\text { Factores } \\
\text { condicionantes }\end{array}$ & $\begin{array}{l}\text { Salud y } \\
\text { educación } \\
\text { básica }\end{array}$ & $\begin{array}{l}\text { Infraestructura } \\
\text { (infraestructura } \\
\text { básica, infraestructura } \\
\text { tecnológica, } \\
\text { infraestructura científica, } \\
\text { salud y medo ambiente, } \\
\text { educación) }\end{array}$ & $\begin{array}{l}\text { Flujos de } \\
\text { capital e } \\
\text { inversión } \\
\text { extranjera }\end{array}$ & $\begin{array}{l}\text { Capital } \\
\text { mínimo } \\
\text { necesario }\end{array}$ \\
\hline \multirow[t]{4}{*}{ Gobierno } & $\begin{array}{l}\text { Educación } \\
\text { superior y } \\
\text { capacitación }\end{array}$ & & $\begin{array}{l}\text { Salarios y } \\
\text { precios }\end{array}$ & \\
\hline & $\begin{array}{l}\text { Eficiencia del } \\
\text { mercado }\end{array}$ & & $\begin{array}{l}\text { Derechos de } \\
\text { propiedad }\end{array}$ & \\
\hline & Tecnología & & $\begin{array}{l}\text { Marco } \\
\text { regulatorio }\end{array}$ & \\
\hline & $\begin{array}{l}\text { Estrategia y } \\
\text { operaciones de } \\
\text { la empresa }\end{array}$ & & $\begin{array}{l}\text { Actividad } \\
\text { del mercado } \\
\text { informal }\end{array}$ & \\
\hline
\end{tabular}




\section{b) Factores sectoriales}

Desde la perspectiva donde los factores que pueden definir la rentabilidad y competitividad en las empresas son los sectores industriales, y no las naciones, existen diferentes autores y modelos que a través de los últimos veinte años han venido hacer aportaciones en este sentido. En este enfoque, Jaquemin (1982), por ejemplo, ha propuesto explicaciones a la competitividad de las empresas que se derivan de las características de los mercados en los que éstas operan. En este sentido, el modelo de Jaquemin viene a considerar a la industria como una unidad homogénea, donde las empresas que la constituyen son semejantes en aquellas variables económicas importantes, excepto en todo caso en su tamaño, lo cual permite la aparición de un cierto poder de mercado (Cabral, 1997).

Por otro lado, existen diferentes corrientes teóricas que han afirmado al respecto que la estructura y configuración organizativa de un sector termina por ejercer una fuerte influencia sobre la competitividad de las empresas, ya que dicha estructura determina las reglas del juego competitivo y, por ende, las posibilidades estratégicas de las empresas (Porter, 1991). Bueno (1992), en este mismo sentido, agrega en su modelo de competitividad denominado "Modelo de las diez fuerzas" que además del poder explicativo de la estructura del sector también existen diferentes elementos que explican la competitividad de aquellas empresas que justamente pertenecen a ese mismo sector; él las denomina como poderes de negociación de clientes, proveedores, público, propietarios y del tipo social. En el cuadro 2 se puede observar un comparativo de modelos en el nivel de sector industrial. 


\section{Cuadro 2}

\section{Comparativo de modelos de competitividad en el nivel sector}

\begin{tabular}{|c|c|c|}
\hline Jacquemin (1982) & Porter (1982 y 1987) & Bueno (1991) \\
\hline $\begin{array}{l}\text { 1. Diferenciación de } \\
\text { productos. } \\
\text { 2. Barreras de entrada. } \\
\text { 3. Grado de concentración. } \\
\text { 4. Naturaleza de la demanda. }\end{array}$ & $\begin{array}{l}\text { 1. Competidores actuales en el } \\
\text { sector industrial. } \\
\text { 2. Competidores potenciales } \\
\text { 3. Productos sustitutivos } \\
\text { 4. Poder de negociación de los } \\
\text { clientes } \\
\text { 5. Poder negociador de los } \\
\text { proveedores. }\end{array}$ & $\begin{array}{l}\text { 1. Nuevos competidores. } \\
\text { Nuevas empresas. } \\
\text { Competencia internacional. } \\
\text { Competencia de otros sectores. } \\
\text { 2. Nuevos productos. } \\
\text { Productos sustitutivos. } \\
\text { 3. Poder negociador de los clientes. } \\
\text { 4. Poder de negociación de los } \\
\text { proveedores. } \\
\text { 5. Poder de negociación de la } \\
\text { Administración Pública (poder } \\
\text { público). } \\
\text { 6. Poder de negociación de los } \\
\text { propietarios (poder económico). } \\
\text { 7. Poder de negociación de los } \\
\text { sindicatos, organizaciones } \\
\text { políticas, asociaciones de } \\
\text { consumidores (poder social). }\end{array}$ \\
\hline
\end{tabular}

Fuente: Bueno (1994). Curso Básico de economía de la empresa

\section{c) Factores empresariales}

Actualmente, se viene manifestando una progresiva toma de conciencia a favor de la consideración de los factores más propios de la empresa como las variables claves de su competitividad. En palabras de Fernández (1992, p. 32):

La competitividad de una empresa no está determinada de forma inequívoca por las características del territorio en que actúe o de los sectores a los que formalmente pertenezca. La capacidad de una empresa para tener éxito en mercados cada vez más grandes, abiertos y competitivos depende, sobre todo, de ella misma

La competitividad de las empresas, entonces, debe tener un componente que se fundamenta en los conocimientos y habilidades que domina la organización, entre los que se incluye su sistema de gestión. Para Cuervo (1993), el análisis sectorial es relevante en algunos casos, pero no determina el margen de beneficios de las 
empresas, debiéndose entender la competitividad misma de la empresa a partir de sus propios recursos y habilidades más que en las características estructurales de los sectores.

Además hay que entender que las empresas no son agentes homogéneos que practican un mismo tipo de comportamiento, sino que son organizaciones con estructuras internas complejas que definen una diferente personalidad y que actúan en situaciones de incertidumbre con una limitada capacidad de racionalidad en el intento de conseguir los distintos objetivos que se han establecido como respuesta al conflicto de intereses de sus individuos participantes (Huerta, 1993).

En el cuadro 3 se describen los factores empresariales que, según diversos autores, son los más comúnmente señalados como determinantes de la competitividad empresarial.

\section{Cuadro 3}

Factores empresariales determinantes de la competitividad según diversos autores

\begin{tabular}{l|l}
\hline Autor & Factores determinantes \\
\hline $\begin{array}{l}\text { Maidique y Patch } \\
\text { (1978) }\end{array}$ & $\begin{array}{l}\text { Las estrategias de mercado, la preferencia de } \\
\text { consumidores y la especialización del producto. }\end{array}$ \\
\hline Serralde (1997) & $\begin{array}{l}\text { La participación relativa en el mercado, la calidad de } \\
\text { los productos y servicios, la rentabilidad, la cobertura } \\
\text { de los canales de distribución, la reputación de los } \\
\text { productos, la fuerza de la investigación y desarrollo, } \\
\text { las relaciones con el gobierno. }\end{array}$ \\
\hline Wilensky (1996) & $\begin{array}{l}\text { El precio, el producto, la promoción, la plaza y la } \\
\text { posventa. }\end{array}$ \\
\hline Gutiérrez (1999) & $\begin{array}{l}\text { Calidad, el precio y el tiempo de entrega de sus } \\
\text { productos y servicios. }\end{array}$ \\
\hline Álvarez (1999) & Ventas, utilidad y participación de mercado. \\
\hline García (1999) & Calidad, calidad del servicio, relaciones públicas. \\
\hline Hernández y & $\begin{array}{l}\text { Satisfacción del cliente, calidad del producto, } \\
\text { utilidades (ingresos). }\end{array}$ \\
\hline Rodríguez (2000) & $\begin{array}{l}\text { Calidad, precio, tecnología, capacitación y los canales } \\
\text { de distribución. }\end{array}$ \\
\hline Bonales (2001)
\end{tabular}

\section{Descripción del sector industrial de telefonía móvil en México}

A pocos años de haberse efectuado la primera llamada por teléfono celular, el número de aparatos móviles de comunicación ha rebasado hasta en un trescientos por ciento la cantidad total de teléfonos fijos instalados en el país. Según datos 
aportados por la Comisión Federal de Telecomunicaciones, en 1989 sólo había 64,000 usuarios del servicio celular, mientras que en junio del 2010 el número superó los 80 millones de usuarios.

Una de las principales características del sector de la telefonía móvil de México ha sido su dinamismo permanente, reflejado principalmente en el acelerado cambio tecnológico necesario para la adopción de los nuevos e innovadores servicios ofrecidos por las empresas pertenecientes a este sector, lo que a su vez ha provocado amplios niveles de inversión de dichas empresas, tanto en términos de infraestructura tecnológica como en términos de gastos de concesión para la explotación del servicio. Por lo tanto, es frecuente que, al reseñar el mercado móvil en México, se le refiera como ampliamente exitoso, en donde el crecimiento, la rentabilidad y la innovación tecnológica han sustentado los buenos resultados del sector durante los últimos años.

Sin embargo, este crecimiento en México se ha visto acompañado de un alto nivel de concentración de mercado, pues en diciembre de 2009 la empresa dominante del sector cubrió el 73.8\% de participación del mismo, dejando a sus demás competidoras con el siguiente orden de participación: Telefónica Movistar con 21.6\% y Iusacell Unefón con un 4.4\%, que se fusionaron desde 2007 (ver cuadro 4).

\section{Cuadro 4}

Participación del mercado en el sector móvil mexicano a diciembre de 2009

\begin{tabular}{c|c}
\hline Empresa & Participación de mercado \\
\hline Telcel & $73.8 \%$ \\
\hline Telefónica Movistar & $21.6 \%$ \\
\hline Iusacell Unefón & $4.4 \%$ \\
\hline
\end{tabular}

Paralelo a este nivel de concentración, el éxito competitivo ${ }^{1}$ de los diferentes actores también ha presentado diversas discontinuidades a través de todos estos años, que van desde altos rendimientos económicos, por un lado, hasta niveles negativos de rendimiento, por otro.

\footnotetext{
${ }^{1}$ El logro del éxito competitivo se manifiesta en la obtención por parte de la empresa de rentas superiores a las obtenidas por sus competidores. En este sentido se entiende por renta aquellos ingresos que exceden el coste de oportunidad de los recursos propios (Mahoney y Pandian. 1992).
} 
Es decir, los resultados obtenidos por las empresas operadoras de este sector en México difieren, en gran medida, de una respecto a las otras, ${ }^{2}$ lo que en la práctica hace que las rigurosas condiciones impuestas por el modelo teórico de competencia perfecta no se cumplan cuando menos en el sector móvil mexicano. ${ }^{3}$

Como ejemplo, se pueden citar las diferencias que se presentaron en el nivel de ingresos entre la empresa más rentable del sector (Telcel) y las demás. En el cuadro 5 se observan estas diferencias, donde, por ejemplo, al cierre del año 2009 Telcel presentó ingresos por 142,362 millones de pesos en contraste con los 11,145 millones de pesos presentados por Iusacell-Unefón en este mismo periodo.

\section{Cuadro 5}

\section{Resultados de operadoras en términos rentables 2002-2009 (millones de pesos)}

\begin{tabular}{|c|c|c|c|c|c|}
\hline \multicolumn{2}{|c|}{$\begin{array}{c}\text { Resultados competitivos } \\
\text { 2002-2006 }\end{array}$} & \multirow{2}{*}{$\begin{array}{l}\text { Telcel } \\
44,608\end{array}$} & \multirow{2}{*}{$\begin{array}{c}\text { Movistar } \\
6,975\end{array}$} & \multirow{2}{*}{$\begin{array}{c}\text { Iusacell } \\
6,297\end{array}$} & \multirow{2}{*}{$\begin{array}{r}\text { Unefón } \\
3,159\end{array}$} \\
\hline \multirow{8}{*}{$\begin{array}{c}\text { Total de } \\
\text { ingresos } \\
\text { (millones de } \\
\text { pesos) }\end{array}$} & 2002 & & & & \\
\hline & 2003 & 57,027 & 8,100 & 4,962 & 4,229 \\
\hline & 2004 & 73,180 & 10,622 & 5,398 & 4,437 \\
\hline & 2005 & 89,586 & 11,685 & 6,770 & 4,005 \\
\hline & 2006 & 110,055 & 14,820 & 8,153 & \multirow{4}{*}{ ND } \\
\hline & 2007 & 124,391 & \multirow{3}{*}{ ND } & 10,273 & \\
\hline & 2008 & 135,278 & & 11,090 & \\
\hline & 2009 & 142,362 & & 11,145 & \\
\hline \multirow{8}{*}{$\begin{array}{c}\text { Utilidad } \\
\text { operativa } \\
\text { (millones de } \\
\text { pesos) }\end{array}$} & 2002 & 12,928 & -220 & $-2,328$ & 587 \\
\hline & 2003 & 19,234 & $-1,620$ & $-4,832$ & 1,086 \\
\hline & 2004 & 26,388 & $-1,620$ & $-1,977$ & 1,300 \\
\hline & 2005 & 34,413 & $-2,385$ & -685 & 1,333 \\
\hline & 2006 & 48,693 & -150 & -207 & \multirow{4}{*}{ ND } \\
\hline & 2007 & 58,372 & \multirow{3}{*}{ ND } & -68 & \\
\hline & 2008 & 62,459 & & 379 & \\
\hline & 2009 & 69,154 & & ND & \\
\hline
\end{tabular}

Por lo que respecta al margen de utilidad operativa, de acuerdo con el cuadro 5, la diferencia también fue marcada entre estas empresas, pues Telcel presentó utili-

\footnotetext{
${ }^{2}$ Las empresas operadoras son aquellas que ofrecen el servicio de telefonía móvil como Telcel, Iusacell, Movistar y Unefón.

${ }^{3}$ Cuando los participantes se encuentran inmersos en condiciones de competencia perfecta se espera que las ganancias obtenidas por el conglomerado de empresas sean repartidas entre cada uno de los operadores de manera proporcional a su poder de mercado, el cual debe ser el más equilibrado posible (Bueno, 1996).
} 
dades positivas en los últimos años, llegando incluso en el 2009 a 69,154 millones de pesos, mientras que sus competidoras presentaron pérdidas en algunos años inclusive. Algo parecido ocurrió cuando se compararon datos de competitividad con criterio de posición de mercado. En el cuadro 6 se observan también las marcadas diferencias entre el líder y seguidores en términos de número de clientes. Resalta en este cuadro, por ejemplo, las diferencias entre Telcel en el año 2009 con sus 59.2 millones de usuarios contra los 17.4 millones de Telefónica y los 3.59 millones de clientes de Iusacell Unefón. En el cuadro 6 también se refleja la consolidación de usuarios que a partir del año 2007 Iusacell y Unefón llevaron a cabo después de su fusión.

\section{Cuadro 6}

\section{Resultados de las operadoras en términos de crecimiento 2002-2009}

\begin{tabular}{|c|c|c|c|c|c|}
\hline \multicolumn{2}{|c|}{$\begin{array}{c}\text { Resultados competitivos } \\
\text { 2002-2006 }\end{array}$} & \multirow{2}{*}{$\begin{array}{c}\text { Telcel } \\
77 \% \\
\end{array}$} & \multirow{2}{*}{$\begin{array}{c}\text { Movistar } \\
15 \%\end{array}$} & \multirow{2}{*}{$\begin{array}{c}\text { Iusacell } \\
4 \%\end{array}$} & \multirow{2}{*}{$\begin{array}{c}\text { Unefon } \\
3 \%\end{array}$} \\
\hline \multirow{7}{*}{$\begin{array}{l}\text { Posición de } \\
\text { mercado }\end{array}$} & 2003 & & & & \\
\hline & 2004 & $75 \%$ & $18 \%$ & $4 \%$ & $3 \%$ \\
\hline & 2005 & $78 \%$ & $14 \%$ & $4 \%$ & $4 \%$ \\
\hline & 2006 & $78 \%$ & $16 \%$ & $4 \%$ & $2 \%$ \\
\hline & 2007 & $75.7 \%$ & $18.1 \%$ & \multicolumn{2}{|c|}{$6 \%$} \\
\hline & 2008 & $74.9 \%$ & $20.3 \%$ & \multicolumn{2}{|c|}{$4.6 \%$} \\
\hline & 2009 & $73.8 \%$ & $21.6 \%$ & \multicolumn{2}{|c|}{$4.4 \%$} \\
\hline \multirow{7}{*}{$\begin{array}{l}\text { Millones de } \\
\text { suscriptores }\end{array}$} & 2003 & 23.4 & 4.2 & 1.27 & 1.2 \\
\hline & 2004 & 28.8 & 6.7 & 1,46 & 1.4 \\
\hline & 2005 & 35.9 & 6.4 & 1.85 & 1.4 \\
\hline & 2006 & 43.2 & 8.5 & 2.1 & 1.3 \\
\hline & 2007 & 50.0 & 12 & \multicolumn{2}{|c|}{4.040} \\
\hline & 2008 & 56.3 & 15.3 & \multicolumn{2}{|c|}{3.50} \\
\hline & 2009 & 59.2 & 17.4 & \multicolumn{2}{|c|}{3.59} \\
\hline
\end{tabular}

Desde esta tendencia, la Comisión Federal de Telecomunicaciones ${ }^{4}$ (Cofetel) dictaminó desde 1997 nuevas regulaciones en materia de licitación de servicios inalámbricos con el fin de establecer un marco competitivo; sin embargo, la escala de concentración de mercado se ha mantenido desde aquel año casi sin variación.

\footnotetext{
${ }^{4}$ La Cofetel es un órgano administrativo desconcentrado de la Secretaría de Comunicaciones y Transportes y está encabezada por cuatro comisionados nombrados por el Secretario de Comunicaciones y Transportes en representación del presidente de la República, uno de los cuales ocupa el cargo de presidente de la Cofetel. El objeto de la Cofetel consiste en regular el sector nacional de las telecomunicaciones de México.
} 


\section{Factores explicativos de la competitividad en las empresas de telefonía móvil de México}

Los factores de índole macroeconómico sectorial o incluso empresarial podrían explicar la diferencia de resultados competitivos en las empresas de este sector; por lo tanto, la identificación de factores que condicionan la competitividad en estas empresas adquiere mayor complejidad al reconocer la influencia que sobre ella ejercen distintos agentes del tipo económico, gubernamental, estructural, estratégico y directivo, factores que, por un lado, rodean el ambiente de estas empresas y que, por otro, las caracterizan (Martín, 1993; Montes y Ruiz, 1993; Rodríguez, 1993).

Para el caso de la presente investigación, con base en una consulta a expertos del sector de telefonía móvil, ${ }^{5}$ se logró identificar los cinco factores que, según los expertos, han impactado en un mayor grado la competitividad de las empresas de estudio. Estos factores son: recursos tangibles, tecnología, precio, calidad y productividad (cuadro 7)

\section{Cuadro 7}

Factores de competitividad en las empresas de telefonía móvil de México

\begin{tabular}{c|c|c}
\hline Factor & Autores & Teoría/modelo \\
\hline $\begin{array}{c}\text { Recursos } \\
\text { tangibles }\end{array}$ & $\begin{array}{c}\text { Barney (1991),Grant (1991), } \\
\text { Hammel y Prahalad (1990), Galán } \\
\text { y Vecino (1996), Teece, Pisano y } \\
\text { Shuen (1991), Peteraf (1993) }\end{array}$ & $\begin{array}{c}\text { Recursos y } \\
\text { capacidades }\end{array}$ \\
\hline Tecnología & $\begin{array}{c}\text { Hammel y Prahalad (1990), Galán } \\
\text { y Vecino (1996), Peteraf (1993), } \\
\text { Teece, Pisano y Shuen (1991) }\end{array}$ & MIT \\
\hline Precio & $\begin{array}{c}\text { Wilenski (1997), Sipper y Bulfin } \\
\text { (1999). }\end{array}$ & $\begin{array}{c}\text { Claves de la } \\
\text { competitividad }\end{array}$ \\
\hline Calidad & $\begin{array}{c}\text { Sipper y Bulfin (1999), Gutiérrez } \\
\text { (1999), Jurán (1995). }\end{array}$ & $\begin{array}{c}\text { La rueda de la } \\
\text { competitividad }\end{array}$ \\
\hline Productividad & Sipper y Bulfin (1999), Hitt (2001). & $\begin{array}{c}\text { La rueda de la } \\
\text { competitividad }\end{array}$ \\
\hline
\end{tabular}

\footnotetext{
${ }^{5}$ La consulta a expertos se realizó por medio de un cuestionario individual que fue aplicado a 14 de ellos, entre las que destacó personal técnico y administrativo de dos empresas operadoras de telefonía móvil, además de algunos líderes de proyectos de empresas extranjeras proveedoras de telecomunicaciones. Para ello, se utilizó el índice de concordancia de Kendall con el fin de fundamentar mediante un método no paramétrico dicha selección de factores de competitividad.
} 


\section{Objetivo de investigación}

Determinar un modelo que explique la medida en que los recursos tangibles, el precio, la calidad, la productividad y la tecnología impactan la competitividad de las empresas operadoras de telefonía móvil de México, expresada ésta en términos de rentabilidad y posición de mercado.

\section{Método}

La investigación se concretó con un método hipotético deductivo con un corte transversal y explicativo, pues sólo se tomaron en consideración datos con una consistencia lógica comparable (de 2002 a 2006). La investigación se desarrolló en cuatro fases:

a) Se realizó un análisis de tipo cualitativo para que expertos en el tema discriminaran los factores que a su juicio determinan la competitividad de las empresas de telefonía móvil. Estas variables también coinciden con las aportaciones hechas por Porter (1991), Grant (1996), Barney (1991) y Hammel y Prahalad (1990) respecto a los factores que determinan la competitividad, las que fueron analizadas en el marco teórico antes presentado.

b) Fue formulada la hipótesis principal de la investigación con base en el objetivo principal.

c) Se realizó un análisis cuantitativo de tipo correlacional y explicativo para establecer las relaciones de los factores con la competitividad. Para ello, fue necesario, en primer lugar, diseñar un cuestionario de recolección de datos que fue aplicado a los sujetos de investigación seleccionados. El análisis cuantitativo de la información se hizo por medio de diferentes métodos multivariados, como el factor análisis y el análisis de regresión lineal múltiple, además del estadístico de correlación de Pearson.

d) Finalmente, por medio de los resultados obtenidos del análisis, se contrastó empíricamente la hipótesis formulada, que permitió presentar el modelo causal de competitividad en estas empresas. 


\section{Hipótesis general}

La competitividad de las empresas operadoras del sector de telefonía móvil de México - medida en términos de rentabilidad y posición de mercado- es explicada por sus recursos tangibles, el precio de sus servicios, la calidad ofrecida, su productividad y por su tecnología.

En el diagrama ex ante (esquema 1) se describen las cinco variables independientes y la única dependiente en la investigación; a su vez, también se muestran las dos dimensiones de la variable dependiente: rentabilidad y posición de mercado.

\section{Esquema 1}

Diagrama ex ante

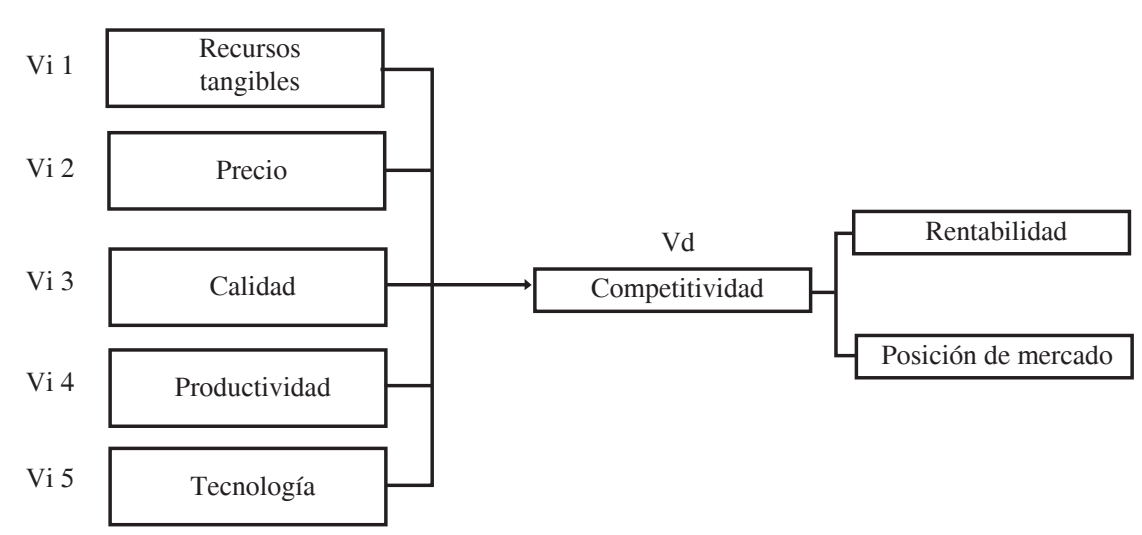

\section{Universo de estudio}

El planteamiento de este trabajo se sustentó en el análisis de cuatro empresas pertenecientes al entorno de la telefonía móvil en México con el objeto de poner de manifiesto aquellos aspectos que puedan identificar de mejor manera los resultados competitivos de cada una de ellas. En este sentido, el universo de estudio contempló las siguientes empresas: Telcel, Movistar, Iusacell y Unefón.

La muestra que se utilizó para la realización de esta investigación fue de tipo no probabilística y predeterminada debido a que el instrumento de investigación se diseñó de tal manera que sólo el personal clave de cada empresa podía entregar y validar cada respuesta solicitada. Por ello, se optó por sujetos de investigación con el siguiente perfil: 
- Pertenecer al staff directivo de la empresa operadora.

- Contar con un alto de nivel de autorización para acceder y entregar la información requerida.

- Contar con la disponibilidad suficiente en tiempo para concentrar todas las respuestas de cada uno de los reactivos solicitados por el instrumento.

- Tener la accesibilidad para ser entrevistado personalmente, al menos un par de veces, además de poder ser contactado telefónicamente en caso de requerirse.

Con base en este perfil, se contactó de manera personal a un ejecutivo por cada una de las empresas operadoras involucradas en el estudio.

\section{Instrumento de recolección}

El instrumento se construyó a partir de la definición de 14 diferentes preguntas, cuyas respuestas permitieron obtener la mayor parte de los indicadores requeridos para la estimación de las variables consideradas según la revisión teórica planteada anteriormente. Los restantes indicadores se obtuvieron a través de las fuentes secundarias de información antes referidas.

El cuestionario está dividido en seis apartados y en total presenta catorce preguntas cerradas como se menciona a continuación:

\section{a) Recursos tangibles}

Indicar en pesos mexicanos las cantidades correspondientes a los ejercicios contables 2002, 2003, 2004, 2005 y 2006:

1.

\begin{tabular}{|l|l|l|l|l|l|}
\hline \multicolumn{2}{c}{2002} & 2003 & 2004 & 2005 & 2006 \\
\hline Propiedades totales no tecnológicas & $\$$ & $\$$ & $\$$ & $\$$ & $\$$ \\
\hline
\end{tabular}

2.

\begin{tabular}{|l|l|l|l|l|l|}
\multicolumn{2}{c}{2002} & 2003 & 2004 & 2005 & 2006 \\
\hline Total de capital contable & $\$$ & $\$$ & $\$$ & $\$$ & $\$$ \\
\hline
\end{tabular}


3.

\begin{tabular}{|l|l|l|l|l|l|}
\multicolumn{1}{c}{2002} & 2003 & 2004 & 2005 & 2006 \\
\hline Total de pasivos & $\$$ & $\$$ & $\$$ & $\$$ & $\$$ \\
\hline
\end{tabular}

\section{b) Precio}

4. Indicar el valor en pesos mexicanos de los precios de los servicios ofrecidos en términos de planes de pospago para cada año.

\begin{tabular}{|l|l|l|l|l|l|}
\multicolumn{2}{c}{2002} & 2003 & 2004 & 2005 & 2006 \\
\hline Renta mensual promedio & $\$$ & $\$$ & $\$$ & $\$$ & $\$$ \\
\hline
\end{tabular}

5. Indicar el valor en pesos mexicanos de los precios de los servicios ofrecidos en términos de planes de prepago para cada año.

\begin{tabular}{|l|l|l|l|l|l|}
\multicolumn{2}{c}{2002} & 2003 & 2004 & 2005 & 2006 \\
\hline $\begin{array}{l}\text { Precio promedio por minuto de voz } \\
\text { a teléfono fijo o móvil }\end{array}$ & $\$$ & $\$$ & $\$$ & $\$$ & $\$$ \\
\hline
\end{tabular}

6. Indicar el valor en pesos mexicanos de los precios de los servicios ofrecidos en términos de mensajes escritos para cada año.

\begin{tabular}{|c|c|c|c|c|c|}
\hline & & 2003 & 2004 & 05 & 2006 \\
\hline $\begin{array}{l}\text { Precio promedio por mensaje escrito } \\
\text { enviado }\end{array}$ & $\$$ & $\$$ & $\$$ & $\$$ & $\$$ \\
\hline
\end{tabular}

\section{c) Calidad}

7. Indicar con una "X" si la empresa tiene implantado el sistema de calidad ISO 9000. Sólo seleccione una opción.

\begin{tabular}{|c|l|}
\hline No Cuenta & \\
\hline Sí Cuenta & \\
\hline
\end{tabular}




\section{d) Productividad}

8. Indicar el número total de usuarios y empleados para cada año solicitado.

\begin{tabular}{|c|c|c|c|c|}
\hline & 2003 & 2004 & 2005 & 2006 \\
\hline Número total de usuarios & & & & \\
\hline Número total de empleados & & & & \\
\hline $\begin{array}{l}\text { Total de usuarios / Total de } \\
\text { empleados }\end{array}$ & & & & \\
\hline
\end{tabular}

9. Indicar el número total de usuarios y espectro de frecuencia en mega hertz concesionado por su empresa según cada año solicitado:

\begin{tabular}{|l|l|l|l|l|l|}
\hline \multicolumn{2}{l}{2002} & 2003 & & & \\
\hline Número total de usuarios & & & & & \\
\hline Espectro concesionado & & & & & \\
\hline $\begin{array}{l}\text { Total de usuarios/espectro } \\
\text { concesionado }\end{array}$ & & & & & \\
\hline
\end{tabular}

\section{e) Tecnología}

10. Indicar en millones de pesos las cantidades correspondientes de gastos incurridos en inversión de planta telefónica para cada año solicitado.

\begin{tabular}{|l|l|l|l|l|l|}
\multicolumn{1}{c}{2002} & 2003 & 2004 & \multicolumn{2}{c}{2005} \\
\hline Inversión en planta telefónica & $\$$ & $\$$ & $\$$ & $\$$ & $\$$ \\
\hline
\end{tabular}

11. Indicar en millones de pesos las cantidades correspondientes en inversión en nuevas tecnologías en los años 2002, 2003, 2004, 2005 y 2006:

\begin{tabular}{|l|l|l|l|l|l|}
\multicolumn{1}{c}{2002} & 2003 & 2004 & 2005 & 2006 \\
\hline $\begin{array}{l}\text { Inversión en nuevas } \\
\text { tecnologías. }\end{array}$ & $\$$ & $\$$ & $\$$ & $\$$ & $\$$ \\
\hline
\end{tabular}

12. Indicar en millones de pesos las cantidades correspondientes en inversión en capacitación técnica incurridas en los años 2002, 2003, 2004, 2005 y 2006 :

\begin{tabular}{|l|l|l|l|l|l|}
\multicolumn{2}{l}{$2002 \quad 2003 \quad 2004$} & & & \\
\hline $\begin{array}{l}\text { Inversión en capacitación } \\
\text { técnica }\end{array}$ & & & & & \\
\hline
\end{tabular}




\section{f) Posición de mercado}

13. Indicar el número total de usuarios activados según cada año solicitado:

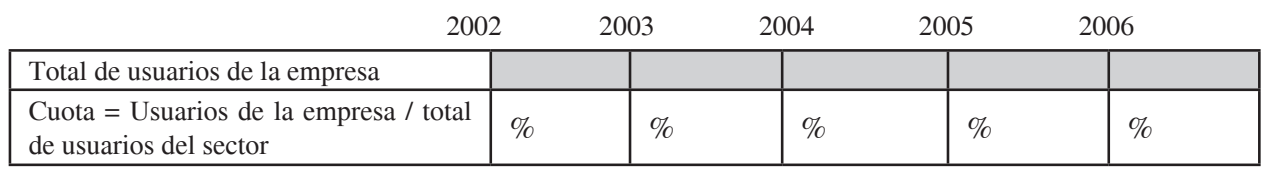

14. Indicar el número de usuarios nuevos que se activaron para cada año solicitado

\begin{tabular}{|l|l|l|l|l|l|}
\multicolumn{2}{l}{2002} & 2003 & & & \\
\begin{tabular}{|l|l|l|l|l|} 
Nuevos usuarios que se activaron de \\
enero a diciembre de cada año
\end{tabular} & & & & & \\
\hline $\begin{array}{l}\text { Crecimiento = Nuevos usuarios/Total } \\
\text { de usuarios de la empresa }\end{array}$ & $\%$ & $\%$ & $\%$ & $\%$ & $\%$ \\
\hline
\end{tabular}

\section{Resultados}

Con base en la simplificación de indicadores de variables por medio de la técnica del análisis factorial de componentes principales, se realizó un segundo nivel de análisis a través de la técnica de regresión múltiple, lo que permitió obtener el modelo causal de competitividad expresado en dos diferentes funciones de regresión: la primera explicó la competitividad de las empresas operadoras de telefonía móvil en términos de rentabilidad; la segunda, la competitividad en términos de posición de mercado.

La primera regresión de la competitividad en rentabilidad se expresa como sigue:

\begin{tabular}{c}
\hline COMPETITIVIDAD_RENT $=0.455$ PRECIO $+0.087 \ldots$ (i) \\
$\mathrm{R}^{2}=0.207$ \\
$\mathrm{R}^{2}$ ajustada $=0.163$ \\
$\mathrm{~F}=4.697$ \\
$\mathrm{DW}=1.167$
\end{tabular}

Se encontró que el coeficiente de regresión del factor explicativo PRECIO fue el único estadísticamente significativo para el modelo de regresión de COMPETITIVIDAD_RENT, lo que significa que la competitividad en términos de rentabilidad varía 0.455 veces cada vez que el factor precio varía en una unidad su valor. El 
modelo de regresión (i) explica sólo en un $16.3 \%$ a la competitividad medida en términos de rentabilidad, ${ }^{6}$ con una $\mathrm{F}$ de Fisher calculada de 4.697 que fue mayor a la obtenida en los cuadros. Con base en este valor de F, el modelo de regresión (i) se considera válido. Con respecto a los factores explicativos recursos tangibles, calidad, productividad y tecnología, éstos se excluyeron del modelo de regresión, pues no se encontró significancia estadística en el análisis (ver anexo I).

La segunda regresión que explica la competitividad en términos de posición de mercado se expresa de la siguiente manera:

$$
\begin{gathered}
\text { COMPETITIVIDAD_POSICIÓN }=0.510 \text { CALIDAD }+0.369 \\
\text { PRODUCTIVIDAD }+0.11 . . \ldots \ldots . . \text { (ii) } \\
\mathrm{R}^{2}=0.634 \\
\mathrm{R}^{2} \text { ajustado }=0.591 \\
\mathrm{~F}=14.701 \\
\mathrm{DW}=1.264
\end{gathered}
$$

Se encontró que los coeficientes de regresión para los factores explicativos CALIDAD y PRODUCTIVIDAD fueron los únicos significativos estadísticamente para la regresión lineal del factor COMPETITIVIDAD POSICIÓN. Con base en los valores de estos coeficientes, se puede afirmar que la competitividad en términos de posición de mercado varía 0.510 veces cada vez el factor calidad varía en una unidad su valor y 0.369 veces cada vez que el factor productividad varía también en una unidad. El modelo de regresión (ii) explicó hasta en un $59.1 \%$ la competitividad medida en términos de posición de mercado, ${ }^{7}$ con una F de Fisher calculada de 14.701 que resultó mayor a la obtenida en los cuadros. Con base en este valor de F, el modelo de regresión (ii) se considera también válido. Con respecto a los factores explicativos recursos tangibles, precio y tecnología, éstos se excluyeron de la regresión dado que no se encontró significancia estadística en el análisis (ver anexo II).

En relación con los factores recursos tangibles y tecnología, al no quedar incluidos finalmente en ninguna de las dos regresiones obtenidas, se puede afirmar que no explican la competitividad de las empresas estudiadas, cuando se considera el efecto combinado de las cinco variables al mismo tiempo.

\footnotetext{
${ }^{6} \mathrm{R}=0.207, \mathrm{R}^{2}$ ajustada $=0.163$.

${ }^{7}$ Considerando el $\mathrm{R}^{2}$ ajustado.
} 
Considerando los dos modelos obtenidos (i y ii), se evidencia que no todas las variables explicativas propuestas vienen a explicar la competitividad de las empresas operadoras móviles de México, sino que más bien sólo tres de estas variables (precio, calidad y productividad) terminan impactando dicha competitividad. A continuación, se presenta esquemáticamente el modelo causal que finalmente explica la competitividad de las empresas operadoras móviles de México.

\section{Esquema 2 \\ Modelo de competitividad obtenido}

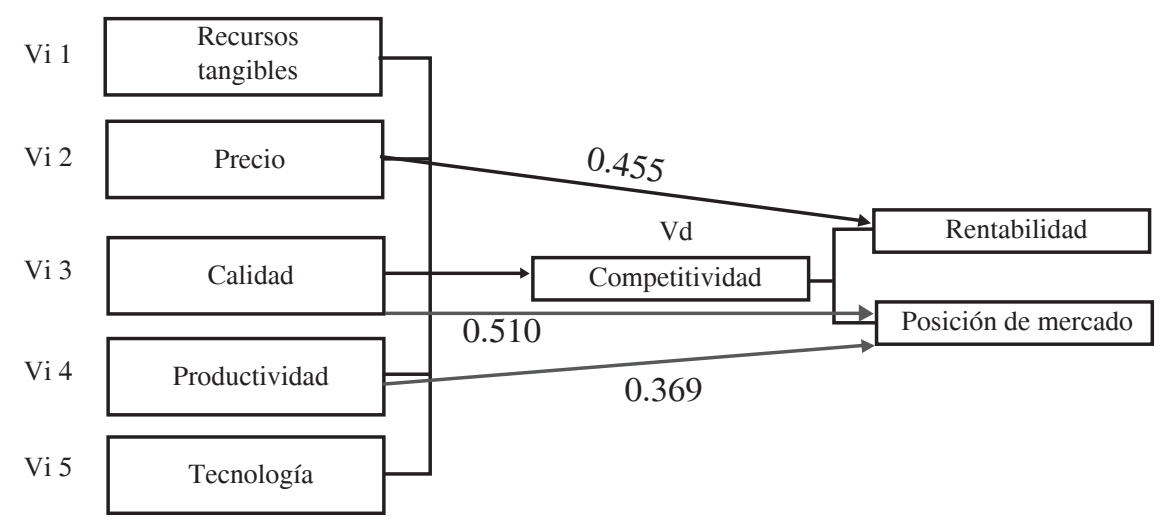

\section{Conclusiones}

En lo referente al cumplimiento del objetivo principal, así como a la hipótesis general de investigación se concluye lo siguiente:

a) El objetivo principal fue alcanzado porque se pudo obtener un modelo compuesto por dos diferentes regresiones estadísticamente significativas. Una para la competitividad medida en términos de rentabilidad y otra para la competitividad medida en términos de posición de mercado. Sin embargo, de acuerdo con el análisis de regresión, el cual analiza el efecto combinado de las variables, sólo el precio, la calidad y la productividad resultaron variables explicativas de la competitividad.

b) Con respecto a la hipótesis general de investigación, que afirmaba que "la competitividad de las empresas operadoras de telefonía móvil de México medida en términos de rentabilidad y posición de mercado es explicada por sus recursos tan- 
gibles, el precio de sus servicios, la calidad ofrecida, su productividad y por su tecnología", y de acuerdo con las dos regresiones lineales obtenidas quedó establecido que ni los recursos tangibles ni la tecnología resultaron factores explicativos de la competitividad, por lo que la hipótesis general de investigación propuesta se contrapone con los hallazgos empíricos de esta investigación.

Con relación a los supuestos teóricos considerados se concluye:

a) En contraposición con los distintos trabajos empíricos, como el desarrollado por Wilenski (1997), se puede afirmar que el precio en el caso de las empresas del sector de telefonía móvil de México no necesariamente debe ser bajo para obtener mejores márgenes de competitividad, pues de acuerdo con los resultados obtenidos del análisis de regresión, en poco o casi nada han contribuido las políticas de bajo precio para la obtención de una mejor posición competitiva dentro de su sector comercial, al menos durante el periodo del año 2002 al 2006. Es decir, con base en Wilenski (1997), a medida que las empresas simplifican los precios de sus servicios, el nivel de rentabilidad y posicionamiento debían aumentar; pero en el sector de telefonía móvil en particular, los bajos precios que ha manejado la empresa Iusacell y Unefón para posicionarse en el mercado no han sido una estrategia exitosa porque su posicionamiento no supera el $4.4 \%$ de preferencia del mercado. Por el contrario, la obtención de grandes rentas y márgenes operativos en estas empresas se ha basado en políticas de sobredimensionamiento en precios que los líderes del sector han impuesto a través de los años.

b) Por otra parte, el hallazgo del factor calidad, como factor explicativo de la competitividad en estas empresas, va en concordancia con las diferentes afirmaciones que asocian a la calidad como un factor determinante de este fenómeno (Crosby, 1991; Juran, 1995; Taguchi, 1999). En este sentido, la mejora de la calidad es un elemento que repetidas veces ha sido considerado por el organismo regulador del sector como un requisito operativo fundamental que todas las empresas que deseen proveer este servicio en México. Esta medida, a la vista de los resultados de la presente investigación, ha sido una medida efectiva que ha llevado a las empresas operadoras a mejorar su competitividad.

c) El tercer factor explicativo de la competitividad en las empresas operadoras fue la productividad, resultado que concuerda con las diferentes afirmaciones presentadas con anterioridad en Hernández (1994) y Sipper y Bulfin (1999). En este sentido, se puede afirmar que aquellas empresas operadoras con altos niveles de 
productividad en el uso de recursos humanos y tecnológicos han sido las que mejores resultados competitivos han alcanzado.

d) Con respecto a los factores recursos tangibles y tecnología, en contraposición con Barney (1991), Grant (1991), Peteraf (1993), Hammel y Prahalad (1990), no resultaron factores explicativos de la competitividad en las empresas estudiadas, lo que permite señalar que las grandes inversiones que realizan estas empresas en la adquisición de tecnología e infraestructura física terminaron por no explicar el nivel de competitividad, esto con base en los modelos (i y ii) previamente mostrados.

\section{Referencias}

Álvarez, T. (1998). Manual de competitividad. México: Editorial Panorama.

Barney, J. (1991). Firm Resources and Sustained Competitive Advantage. Journal of Management 17 (1): 99-120.

Bolsa Mexicana de Valores (2010). Reporte Anual AMX 2009 Disponible en: www.bmv.com.mx

Bueno, E. y P. Morcillo (1994). Fundamentos de economía y organización industrial. Madrid: Mc Graw-Hill.

Cabral, L. (1997). Economía industrial. Madrid: Mc Graw-Hill.

Crosby, P. (1991). La calidad no cuesta. El arte de cerciorase de la calidad. México: CECSA.

Comisión Federal de Telecomunicaciones (2010). Telefonía móvil. Disponible en: www.cft.gob.mx

Cuervo, A. (1993). El papel de la empresa en la competitividad. Papeles de Economía Española (56): 363-378.

Galán, J. L. y J. Vecino (1997). Las fuentes de rentabilidad de la empresa. Revista Europea de Dirección y Economía de la Empresa 6 (1): 21-36. 
García, G. (1993). Esquemas y modelos para la competitividad. México: Ediciones Castillo.

Grant, R.M. (1996). Dirección estratégica: conceptos, técnicas y aplicaciones. Madrid: Civitas.

Gutiérrez, H. (1999). Calidad total y productividad. México: Mc Graw-Hill.

Hamel, G. y C.K. Prahalad (1993). Strategy as Stretch and Leverage, Harvard Business Review, (marzo-abril): 75-84.

Hansen, G. S. y B. Wernerfelt (1989). Determinants of Firm Performance: The Relative Importance of Economic and Organizational Factors. Strategic Management Journal 10: 399- 411.

Hernández, E. (2000). La competitividad industrial en México. México: Plaza y Valdés.

Hernández, S. (1994). Introducción a la administración: Un enfoque teórico práctico. México: Mc Graw-Hill.

Huergo, E. (1992). Tamaño y rentabilidad en la industria española. Economía Industrial (284): 41-49.

Huerta, E. (1993). La empresa: cooperación y conflicto. Madrid: Eudema.

Jacquemin, A. (1982). Economía industrial, estructuras de mercado y estrategias europeas de empresa. Barcelona: Hispano Europea.

Juran, J.M. (1995). Análisis y planeación de la calidad. México: Mc Graw-Hill

Maidique, M. (1978). Corporate strategy and Technological policy. Harvard Business School Case Services. Case 9.

Montes, V. y P. Ruiz (1993). Evolución reciente de la competitividad de la economía y la industria española. Economía Industrial (289): 7-16. 
Peteraf, M. (1993). The Cornestones of Competitive Advantage: A ResourceBased View. Strategic Management Journal 14: 179-191.

Porter, M. (1991). La ventaja competitiva de las naciones. Buenos Aires: Javier Vergara.

Prahalad, C. y G. Hamel (1990). The Core Competence of the Corporation. Harvard Business Review (3): 79-91.

Rumelt, R. (1991). How much does the industry matter? Strategic Management Journal 12: 167-185.

Serralde, A. (1997). Hacia una conciencia de competitividad. Management Today (noviembre).

Schmalensee, R. (1985). Do markets differ much? The American Economic Review 75 (3): 341-351.

Siegel, S. y N. Castellan (2005). Estadística no paramétrica. México: Trillas.

Sipper, D. y R. Bulfin (1999). Planeación y control de la producción. México: Mc Graw-Hill.

Taguchi, G. (1999). Robust Engineering: Learn How to Boost Quality while reducing cost and time to market. USA: Mc Graw-Hill Interamericana.

Teece, D., G. Pisano y Shuen (1990). Firm Capabilities, Resources and Concept of Strategy, Working Paper EAP-38, Universidad de California, pp. 28.

Wilensky, A, (1997). Claves de la estrategia competitiva. Buenos Aires: Fundación OSDE.<smiles></smiles> 


\section{Anexo I}

\section{Análisis de regresión para el resultado con rentabilidad}

\section{Descriptive Statistics}

\begin{tabular}{c|c|c|c}
\hline & Mean & Std. Deviation & $\mathrm{N}$ \\
\hline COM_RENT & .0000000 & 1.00000000 & 20 \\
FAC_TANGIBLES & .0000000 & 1.00000000 & 20 \\
FAC_PRECIO & .0000000 & 1.00000000 & 20 \\
FAC_CALIDAD & .0000000 & 1.00000000 & 20 \\
FAC_PRODUCTIVIDAD & .0000000 & 1.00000000 & 20 \\
FAC_TECNOLOGÍA & .0000000 & 1.00000000 & 20 \\
\hline \multicolumn{2}{|l}{}
\end{tabular}

Model Summary (f)

\begin{tabular}{c|c|c|c|c|c}
\hline Model & $\mathrm{R}$ & R Square & $\begin{array}{c}\text { Adjusted R } \\
\text { Square }\end{array}$ & $\begin{array}{c}\text { Std. Error of the } \\
\text { Estimate }\end{array}$ & Durbin-Watson \\
\hline 1 & $.576(\mathrm{a})$ & .332 & .093 & .95226928 & \\
2 & $.568(\mathrm{~b})$ & .323 & .142 & .92610986 & \\
3 & $.552(\mathrm{c})$ & .305 & .175 & .90835964 & \\
4 & $.524(\mathrm{~d})$ & .275 & .190 & .90014952 & 1.167 \\
5 & $.455(\mathrm{e})$ & .207 & .163 & .91493990 & \\
\hline
\end{tabular}

a Predictors: (Constant), FAC_TECN, FAC_PRECIO, FAC_PRODUCTIVIDAD, FAC_CALIDAD, FAC_ TANGIBLES

b Predictors: (Constant), FAC_PRECIO, FAC_PRODUCTIVIDAD, FAC_CALIDAD, FAC_TANGIBLES

c Predictors: (Constant), FAC_PRECIO, FAC_PRODUCTIVIDAD, FAC_CALIDAD

d Predictors: (Constant), FAC_PRECIO, FAC_PRODUCTIVIDAD

e Predictors: (Constant), FAC_PRECIO

f Dependent Variable: COM_RENT

\begin{tabular}{|c|c|c|c|c|c|c|}
\hline \multicolumn{7}{|c|}{ ANOVA (f) } \\
\hline Model & & $\begin{array}{l}\text { Sum of } \\
\text { Squares }\end{array}$ & df & Mean Square & $\mathrm{F}$ & Sig. \\
\hline 1 & $\begin{array}{l}\text { Regression } \\
\text { Residual } \\
\text { Total }\end{array}$ & $\begin{array}{l}6.305 \\
12.695 \\
19.000\end{array}$ & $\begin{array}{l}5 \\
14 \\
19\end{array}$ & $\begin{array}{l}1.261 \\
.907\end{array}$ & 1.390 & $.287(a)$ \\
\hline 2 & $\begin{array}{l}\text { Regression } \\
\text { Residual } \\
\text { Total }\end{array}$ & $\begin{array}{l}6.135 \\
12.865 \\
19.000\end{array}$ & $\begin{array}{l}4 \\
15 \\
19\end{array}$ & $\begin{array}{l}1.534 \\
.858\end{array}$ & 1.788 & $.184(b)$ \\
\hline 3 & $\begin{array}{l}\text { Regression } \\
\text { Residual } \\
\text { Total }\end{array}$ & $\begin{array}{l}5.798 \\
13.202 \\
19.000\end{array}$ & $\begin{array}{l}3 \\
16 \\
19\end{array}$ & $\begin{array}{l}1.933 \\
.825\end{array}$ & 2.342 & $.112(\mathrm{c})$ \\
\hline 4 & $\begin{array}{l}\text { Regression } \\
\text { Residual } \\
\text { Total }\end{array}$ & $\begin{array}{l}5.225 \\
13.775 \\
19.000\end{array}$ & $\begin{array}{l}2 \\
17 \\
19\end{array}$ & $\begin{array}{l}2.613 \\
.810\end{array}$ & 3.224 & $.065(\mathrm{~d})$ \\
\hline 5 & $\begin{array}{l}\text { Regression } \\
\text { Residual } \\
\text { Total }\end{array}$ & $\begin{array}{l}3.932 \\
15.068 \\
19.000\end{array}$ & $\begin{array}{l}1 \\
18 \\
19\end{array}$ & $\begin{array}{l}3.932 \\
.837\end{array}$ & 4.697 & $.044(e)$ \\
\hline
\end{tabular}


a Predictors: (Constant), FAC_TECN, FAC_PRECIO, FAC_PRODUCTIVIDAD, FAC_ CALIDAD, FAC_TANGIBLES

b Predictors: (Constant), FAC_PRECIO, FAC_PRODUCTIVIDAD, FAC_CALIDAD, FAC_ TANGIBLES

c Predictors: (Constant), FAC_PRECIO, FAC_PRODUCTIVIDAD, FAC_CALIDAD

d Predictors: (Constant), FAC_PRECIO, FAC_PRODUCTIVIDAD

e Predictors: (Constant), FAC_PRECIO

f Dependent Variable: COM_RENT

\section{Anexo II \\ Análisis de regresión para el resultado con posición}

\begin{tabular}{l|l|l|l}
\multicolumn{4}{c}{ Descriptive Statistics } \\
\hline COM_POS & Mean & Std. Deviation & $\mathrm{N}$ \\
FAC_TANGIBLES & .0000000 & 1.00000000 & 20 \\
FAC_PRECIO & .0000000 & 1.00000000 & 20 \\
FAC_CALIDAD & .0000000 & 1.00000000 & 20 \\
FAC_PRODUCTIVIDAD & .0000000 & 1.00000000 & 20 \\
FAC_TECNOLOGÍA & .0000000 & 1.00000000 & 20 \\
\hline
\end{tabular}

\section{Model Summary(e)}

\begin{tabular}{l|l|l|l|l|l}
\hline Model & $\mathrm{R}$ & R Square & $\begin{array}{l}\text { Adjusted R } \\
\text { Square }\end{array}$ & $\begin{array}{l}\text { Std. Error of the } \\
\text { Estimate }\end{array}$ & Durbin-Watson \\
\hline 1 & $.808(\mathrm{a})$ & .653 & .529 & .68604779 & \\
2 & $.808(\mathrm{~b})$ & .653 & .560 & .66300875 & \\
3 & $.802(\mathrm{c})$ & .642 & .575 & .65161669 & \\
4 & $.796(\mathrm{~d})$ & .634 & .591 & .63989400 & 1.264 \\
\hline
\end{tabular}

a Predictors: (Constant), FAC_TECNOLOGÍA, FAC_PRECIO, FAC_PRODUCTIVIDAD, FAC_ CALIDAD,

b Predictors: (Constant), FAC_TECNOLOGÍA, FAC_PRODUCTIVIDAD, FAC_CALIDAD, FAC_TANGIBLES

c Predictors: (Constant), FAC_PRODUCTIVIDAD, FAC_CALIDAD, FAC_TANGIBLES

d Predictors: (Constant), FAC_PRODUCTIVIDAD, FAC_CALIDAD

e Dependent Variable: COM_POS 


\begin{tabular}{|c|c|c|c|c|c|c|}
\hline \multicolumn{7}{|c|}{ ANOVA (e) } \\
\hline Model & & $\begin{array}{l}\text { Sum of } \\
\text { Squares }\end{array}$ & df & Mean Square & $\mathrm{F}$ & Sig. \\
\hline \multirow[t]{3}{*}{1} & Regression & 12.411 & 5 & 2.482 & 5.274 & $.006(a)$ \\
\hline & Residual & 6.589 & 14 & .471 & & \\
\hline & Total & 19.000 & 19 & & & \\
\hline \multirow[t]{3}{*}{2} & Regression & 12.406 & 4 & 3.102 & 7.056 & $.002(b)$ \\
\hline & Residual & 6.594 & 15 & .440 & & \\
\hline & Total & 19.000 & 19 & & & \\
\hline \multirow[t]{3}{*}{3} & Regression & 12.206 & 3 & 4.069 & 9.583 & $.001(\mathrm{c})$ \\
\hline & Residual & 6.794 & 16 & .425 & & \\
\hline & Total & 19.000 & 19 & & & \\
\hline \multirow[t]{3}{*}{4} & Regression & 12.039 & 2 & 6.020 & 14.701 & $.000(d)$ \\
\hline & Residual & 6.961 & 17 & .409 & & \\
\hline & Total & 19.000 & 19 & & & \\
\hline
\end{tabular}

a Predictors: (Constant), FAC_TECNOLOGÍA, FAC_PRECIO, FAC_PRODUCTIVIDAD, FAC_CALIDAD, b Predictors: (Constant), FAC_TECNOLOGÍA, FAC_PRODUCTIVIDAD, FAC_CALIDAD, FAC_ TANGIBLES

c Predictors: (Constant), FAC_PRODUCTIVIDAD, FAC_CALIDAD, FAC_TANGIBLES

d Predictors: (Constant), FAC_PRODUCTIVIDAD, FAC_CALIDAD

e Dependent Variable: COM_POS 